\title{
Performance Analysis of MIMO System over an In-home PLC Channel
}

\author{
Abdelmounim Hmamou ${ }^{1}$, Mohammed EL Ghzaouia ${ }^{2 *}$, Jamal Mestoui ${ }^{2}$ and Jaouad Foshi ${ }^{1}$ \\ ${ }^{\text {I}}$ EEIMP, FST, Moulay Ismail University, Meknes, Morocco \\ ${ }^{2}$ Sidi Mohammed Ben Abdellah University, Faculty of Sciences, Fes, Morocco \\ ${ }^{3}$ ENS, Moulay Ismail University, Meknes, Morocco
}

\begin{abstract}
Power line communication (PLC) system is an attractive technology for Smart Grid applications. One key benefit of PLC is its low installation cost because, in PLC technology, we do not need to install any extra cable to extend a network due to the accessibility to low voltage power network. Orthogonal frequency division multiplexing (OFDM) is widely used in PLC networks. Currently, Multiple Input Multiple Output (MIMO) technology is one of the processing techniques appropriate to PLC networks, allowing high data rate. In this work, the MIMOOFDM system is established to provide better performance over the PLC system by providing communication links with substantial diversity and capacity. However, adapting MIMO to the PLC network involves solving several issues such as MIMO PLC channel modelling and optimisation of the modulation parameters. In this paper, we present measurements results of the transfer function and impulsive noise in the extended frequency range 2-100 MHz. In the simulation part, we evaluate the performance of the proposed receivers in $2 \times 2$ MIMO-PLC channels. It is shown that the minimum mean square error (MMSE) receiver can be one of the appropriate candidates for MIMO PLC channels due to its bite error rate (BER) characteristics under impulsive noise.
\end{abstract}

Keywords: multiple-input and multiple-output (MIMO); orthogonal frequency-division multiplexing (OFDM); power-line communications (PLC); channel capacity; transfer function; bit error rate (BER)

\section{INTRODUCTION}

Considering last-mile communications, Power-line communications (PLC) is a progressive method for data transmission securely. It appears to be an encouraging alternative to conventional technologies such as digital subscriber line (DSL) mainly in rural or underdeveloped areas because it exploits the existing infrastructure. Also, PLC is a suitable applicant for local area networks (LAN) and home automation. Besides, the wireless technologies are strong competitors to the PLC systems. Still, the coexistence of both technologies is expected to be seemed in future communication systems because of their unique characteristics. The PLC system was widely studied in detail by (Llano et al. 2019). However, the PLC channel is a harsh environment for signal transmission. Indeed, the hardness of such a data communication channel can cause performance degradation in the PLC system. On the other side, the data rate and coverage are the biggest challenges in the next generation of PLC networks. One of the most direct and easiest ways to increase both data rate and coverage is to use a Multi-Input Multi-Output (MIMO) system.

Nowadays, the MIMO system has become a favourable technology for high capacity transmission over PLC channels (Shlezinger et al., 2018). MIMO systems can improve data rate by using multiple antennae at the transmitters and the receiver's sides. On the other hand, MIMO systems can offer significant gains in data rates, increase link reliability and

*Corresponding author's e-mail: elghzaoui.mohammed@gmail.com 
power efficiency (Yoon et al., 2012). So, the MIMO system is being there in most modern standards, such as IEEE802.11n and Long-Term Evolution (LTE). Furthermore, the combination of orthogonal frequency division multiplexing (OFDM) and MIMO can be utilised to achieve high diversity gain and high data rates by exploiting spatial multiplexing. For that reason, MIMO-OFDM is one of the most techniques used in developing high-data-rate systems. Hence, MIMOOFDM becomes the dominant technology used for $4 \mathrm{G}$ and future $5 \mathrm{G}$ system (Ertugru, 2016, Jamal et al., 2019). Realising these advantages in practice requires careful exploitation of MIMO-OFDM in PLC networks.

The conventional PLC systems make the use of two cables ( $\mathrm{P}$ and $\mathrm{N}$ ) to connect the transmitter to the receiver. Yet, the indoor electrical wiring has three wires. 3-wire connections essentially allow more feeding and receiving options. For that reason, MIMO PLC systems are envisaged to use the third wire as well (Lars et al., 2014).

The principal behind the MIMO technique over the PLC channel is to use multiple antennas at the transmitter/receiver sides, to increase data rate as well as to overcome channel problems such as noise and multi-path. Indeed, MIMO systems can improve data rate by using multiple antennae at the transmitters and the receiver sides. In effect, MIMO systems use a combination of multiple antennas and multiple signal paths to gain knowledge of the communications channel. By using the spatial dimension of a communications link, MIMO systems can achieve significantly higher data rates than traditional SISO system. However, one of the key tasks that designers of MIMO PLC technology face is reliably transmission information to the receiver in the presence of signal fading. To realise this aim, we investigate the effect of antenna diversity for a MIMO supported PLC system. To do that, we focus on the receiver side by using minimum mean square error (MMSE), maximal ratio combining (MRC) and Equal Gain Combining (EGC) techniques. Moreover, we conducted the measurements of MIMO PLC channel capacity and transfer function on an inhome PLC network.

This work starts by introducing the concept and relevance of MIMO-PLC system, followed by representing ALAMOUTI scheme with $2 \times 2$ diversity order. Measurements results of the transfer function and impulsive noise will be presented:
For example, the channel capacity of SISO and MIMO systems, MIMO and SISO average spectral efficiency $\eta$ and impulsive noise. Another contribution of this paper is the presentation of simulation results comparing MRC, EGC and MMSE receiver in MIMO OFDM PLC channels with Alamouti encoding.

\section{MIMO-OFDM PLC SYSTEM}

The indoor electrical Grids use three wires which allow the use for MIMO PLC technology. The wires are N (Neutral), P (Phase or Live) and PE (Protective Earth). As a result, there are three basic connections, i.e. PE to N, P to N and PE to P. The existing PLC networks use only the P-N possibility to connect the transmitter to the receiver. So, MIMO PLC systems are predicted to make the use of the unused PE wire as well. Nevertheless, according to Kirchhoff's law, we can use only two input feeds simultaneously. At the receiver, all three different receiving ports can be used. In addition, the common-mode (CM) path can also be employed as the fourth receiving port. As shown in Figure1, a $2 \times 2$ MIMO-PLC configuration will be used in this work.

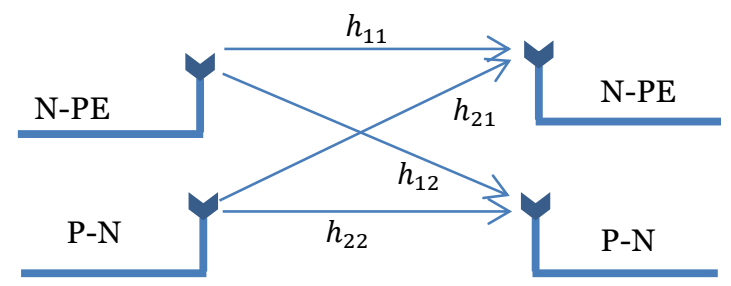

Figure 1. 2x2 MIMO-PLC paths

\section{A. Alamouti Scheme for MIMO-OFDM PLC}

The Alamouti technique may be applied to $2 * 4$ MIMO PLC systems (Lars et al., 2014). The Alamouti scheme works independently of the number of receive ports. Multi-Input Single-Output (MISO) is possible as well. Figure 2 shows the 2*4 MIMO PLC systems.

The MIMO PLC channel system can be represented by: $\mathrm{r}(\mathrm{t})=\mathrm{h}(\mathrm{t}) * \mathrm{~s}(\mathrm{t})+\mathrm{n}(\mathrm{t})$ where $\mathrm{h}(\mathrm{t})$ is the impulse response of the PLC channel, $r(t)$ is the received signal, $n(t)$ is the impulsive noise and $s(t)$ is the transmitted signal.

We start by given the transmitted signal in the general case with Nt transmitters: 


$$
s_{i}(n)\left\{\begin{array}{l}
s_{i}^{\prime}\left(n+N_{s}-N_{C P}\right), \quad n=0, \ldots . N_{C P}-1 \\
s_{i}\left(n-N_{C P}\right), \quad n=N_{C P} \ldots . N_{S}+N_{C P}-1
\end{array}\right.
$$

When combined with SISO-OFDM, implies that

$$
s_{i}(n)=\left\{\begin{array}{l}
\sum_{k=0}^{N_{s}-1} x(k) e^{+\frac{i 2 \pi k\left(n+N_{s}-N_{C P}\right)}{N_{S}}}, n=0, \ldots, N_{c p}-1 \\
\sum_{k=0}^{N_{s}-1} x(k) e^{+\frac{j 2 \pi k\left(n-N_{C P}\right)}{N_{S}}}, n=N_{C P}, \ldots, N_{s}+N_{C P}-1
\end{array}\right.
$$

where $\mathrm{z}_{\mathrm{l}}(\mathrm{n})$ denote the impulsive noise generated by PLC channel at receiver 1 .

$$
\begin{aligned}
& \mathrm{r}_{\mathrm{j}}(\mathrm{n})=\sqrt{\rho} \sum_{\mathrm{j}=1}^{\mathrm{N}_{\mathrm{t}}} \sum_{\mathrm{i}=0}^{\mathrm{L}-1} \mathrm{~h}_{\mathrm{l}, \mathrm{j}}^{\mathrm{i}} \mathrm{s}_{\mathrm{j}}(\mathrm{n}-1)+\mathrm{z}_{\mathrm{l}}(\mathrm{n}) \\
& \mathrm{n}=0, \ldots, \mathrm{N}_{\mathrm{s}}+\mathrm{N}_{\mathrm{CP}}-1
\end{aligned}
$$

The received signal is given by

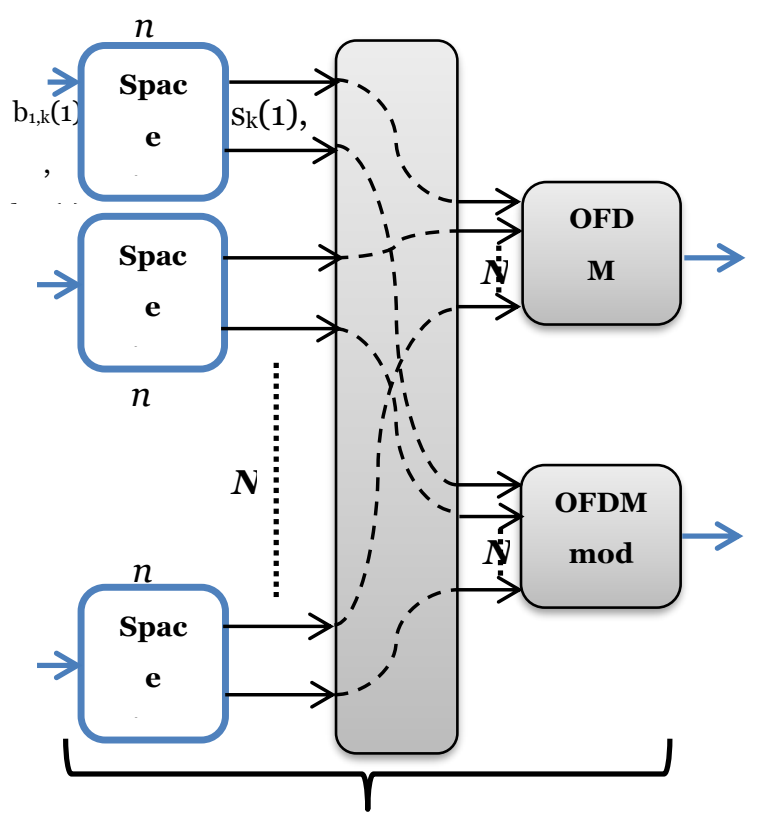

Trans

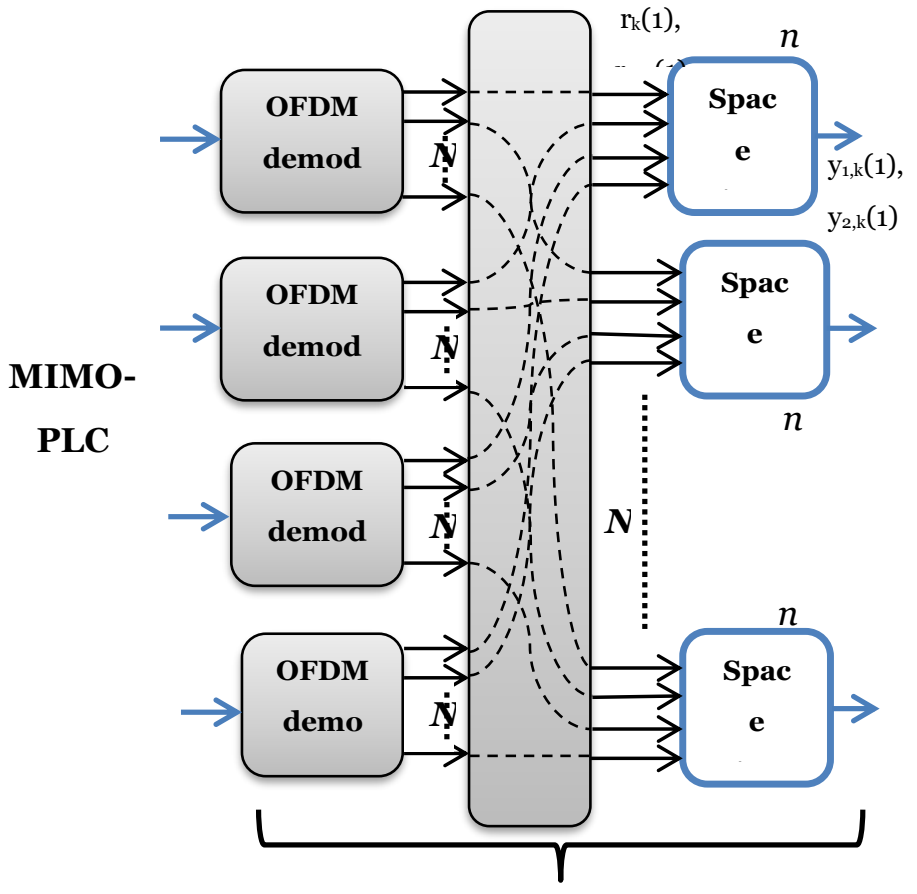

Receive

Figure 2. Block diagram of the MIMO-OFDM Alamouti system, 2×4 MIMO PLC channel

The signal at the receiver is given by;

$$
\mathrm{r}^{\prime} \triangleq\left[\mathrm{r}_{\mathrm{l}}\left(\mathrm{N}_{\mathrm{CP}}\right), \mathrm{r}_{\mathrm{l}}\left(\mathrm{N}_{\mathrm{CP}}+1\right), \ldots, \mathrm{r}_{\mathrm{l}}\left(\mathrm{N}_{\mathrm{s}}+\mathrm{N}_{\mathrm{CP}}-1\right)\right]^{\mathrm{T}}
$$

Thus,

$$
r_{1}^{\prime}(n)= \begin{cases}r_{i}\left(n+N_{C P}\right), & n=0, \ldots, N_{s}-1 \\ 0 & \text { otherwise }\end{cases}
$$

The receiver then performs the DFT;

$$
\begin{array}{r}
r_{j}\left(k^{\prime}\right)=\sum_{n=0}^{N_{s}-1} r_{l}^{\prime}(n) e^{-\frac{2 j \pi k k^{\prime} n}{N_{S}}} \sum_{n=0}^{N_{s}-1} r_{i}\left(n+N_{C P}\right) e^{-\frac{2 j \pi k^{\prime} n}{N_{s}}}(6) \\
r_{j}\left(k^{\prime}\right)=\sqrt{\rho} \sum_{j=1}^{N_{t}} \sum_{i=0}^{L-1} \sum_{k=0}^{N_{s}-1} h_{1, j}^{i} x_{j}(k) e^{\frac{-2 j \pi k i}{N_{S}}}\left(\sum_{n=0}^{N_{s}-1} e^{-\frac{2 j \pi\left(k-k^{\prime}\right) n}{N_{S}}}\right) \\
+Z_{i}\left(k^{\prime}\right), k^{\prime}=0, \ldots, N_{s}-1
\end{array}
$$

(7) can be simplified as follow;

$$
\begin{gathered}
\mathrm{s}: \mathrm{y}_{\mathrm{j}}\left(\mathrm{k}^{\prime}\right)=\mathrm{N}_{\mathrm{s}} \sqrt{\rho} \sum_{\mathrm{j}=1}^{\mathrm{N}_{\mathrm{t}}} \sum_{\mathrm{i}=0}^{\mathrm{L}-1} \sum_{\mathrm{n}}^{\mathrm{N}_{\mathrm{s}}-1} \mathrm{~h}_{\mathrm{l}, \mathrm{j}}^{\mathrm{i}} \mathrm{x}_{\mathrm{j}}(\mathrm{k}) \mathrm{e}^{\frac{-\mathrm{j} 2 \pi \mathrm{ki}}{N_{\mathrm{S}}}} \delta\left(\mathrm{k}-\mathrm{k}^{\prime}\right)+\mathrm{Z}_{\mathrm{i}}\left(\mathrm{k}^{\prime}\right) \\
=\mathrm{N}_{\mathrm{s}} \sqrt{\rho} \sum_{\mathrm{j}=0}^{\mathrm{N}_{\mathrm{t}}} \mathrm{x}_{\mathrm{j}}\left(\mathrm{k}^{\prime}\right) \mathcal{H}_{\mathrm{lj}}\left(\mathrm{k}^{\prime}\right)+\mathrm{Z}_{\mathrm{l}}\left(\mathrm{k}^{\prime}\right), \mathrm{k}^{\prime}=0, \ldots, \mathrm{N}_{\mathrm{s}}-1
\end{gathered}
$$




$$
\mathrm{X}\left(\mathrm{k}^{\prime}\right) \triangleq\left(\begin{array}{c}
\mathrm{x}_{1}\left(\mathrm{k}^{\prime}\right) \\
\mathrm{x}_{2}\left(\mathrm{k}^{\prime}\right) \\
\vdots \\
\mathrm{x}_{\mathrm{N}_{\mathrm{t}}}\left(\mathrm{k}^{\prime}\right)
\end{array}\right)
$$

and

$$
\mathrm{Z}\left(\mathrm{k}^{\prime}\right) \triangleq\left(\begin{array}{c}
\mathrm{Z}_{1}\left(\mathrm{k}^{\prime}\right) \\
\mathrm{Z}_{2}\left(\mathrm{~K}^{\prime}\right) \\
\vdots \\
\mathrm{Z}_{\mathrm{N}_{\mathrm{r}}}\left(\mathrm{k}^{\prime}\right)
\end{array}\right)
$$

\section{B. Alamouti Code in MIMO-PLC System with RMC Receiver}

To understand the concept of Alamouti code in MIMO-PLC system with RMC receiver, we consider the $2 \times 2$ MIMO system. Let hli be the channel gain from transmit antenna i to receive antenna l. we adopt that the received signal is $\mathrm{Z}(\mathrm{t})$, at the first receive where $\mathrm{l}=1$ we can be written;

$$
\mathrm{Z}_{1}=\mathrm{h}_{11}\left(\begin{array}{c}
\mathrm{s}_{1} \\
-\mathrm{s}_{2}^{*}
\end{array}\right)+\mathrm{h}_{12}\left(\begin{array}{c}
\mathrm{s}_{2} \\
\mathrm{~s}_{1}^{*}
\end{array}\right)+\left(\begin{array}{c}
\mathrm{x}_{11} \\
\mathrm{x}_{12}
\end{array}\right)
$$

So $Z_{1}$ can be expressed as fellow

$$
\begin{aligned}
& \mathrm{Z}_{1}=\left(\begin{array}{l}
\mathrm{h}_{11} \mathrm{~s}_{1}+\mathrm{h}_{12} \mathrm{~s}_{2} \\
\mathrm{~h}_{12} \mathrm{~s}_{1}^{*}-\mathrm{h}_{11} \mathrm{~s}_{2}^{*}
\end{array}\right)+\left(\begin{array}{l}
\mathcal{R}_{11} \\
\mathcal{R}_{12}
\end{array}\right)=\mathrm{Gh}_{1}+\mathcal{R}_{1} ; \\
& \mathrm{G}=\left(\begin{array}{ll}
\mathrm{s}_{1} & \mathrm{~s}_{2} \\
-\mathrm{s}_{2}^{*} & \mathrm{~s}_{1}^{*}
\end{array}\right)
\end{aligned}
$$

where $h_{1}=\left(\begin{array}{ll}h_{11} & h_{12}\end{array}\right)^{t}$. Likewise, for $l=2 Z_{2}$ can be expressed as:

$$
\mathrm{Z}_{2}=\mathrm{h}_{21}\left(\begin{array}{c}
\mathrm{s}_{1} \\
-\mathrm{s}_{2}^{*}
\end{array}\right)+\mathrm{h}_{22}\left(\begin{array}{c}
\mathrm{s}_{2} \\
\mathrm{~s}_{1}^{*}
\end{array}\right)+\left(\begin{array}{c}
\mathrm{N}_{21} \\
\mathrm{~N}_{22}
\end{array}\right)
$$

So,

$$
\mathrm{Z}_{2}=\left(\begin{array}{l}
\mathrm{h}_{21} \mathrm{~s}_{1}+\mathrm{h}_{22} \mathrm{~s}_{2} \\
\mathrm{~h}_{22} \mathrm{~s}_{1}^{*}-\mathrm{h}_{21} \mathrm{~s}_{2}^{*}
\end{array}\right)+\left(\begin{array}{l}
\mathcal{R}_{21} \\
\mathcal{R}_{22}
\end{array}\right)=\mathrm{Gh}_{2}+\mathcal{R}_{2} ;
$$

Where $h_{2}=\left(\begin{array}{ll}h_{21} & h_{22}\end{array}\right)^{t}$. The vector $Z_{1}$ can be mapped into the vector $\mathrm{r}_{1}=\mathcal{M}_{l} s+N_{l}$ by altering the second element $Z_{l 2}$ of $Z_{l}=\left(\begin{array}{ll}Z_{l 1} & Z_{l 2}\end{array}\right)^{t}$ into its complex conjugate. Thus, for $s=$ $\left(\begin{array}{ll}s_{1} & s_{2}\end{array}\right)^{t}$ we have

$$
r_{l}=\mathcal{M}_{l} s+N_{l}=\left(\begin{array}{l}
Z_{l 1} \\
Z_{l 2}^{*}
\end{array}\right), \mathrm{l}=1,2
$$

thus

$$
r_{l}=\left(\begin{array}{cc}
h_{11} & h_{12} \\
h_{12}^{*} & -h_{11}^{*}
\end{array}\right)\left(\begin{array}{l}
s_{1} \\
s_{2}
\end{array}\right)+\left(\begin{array}{l}
\aleph_{11} \\
\aleph_{12}
\end{array}\right)
$$

or

$$
r_{1}=\mathcal{M}_{1} s+N_{1}, \quad \mathcal{M}_{1}=\left(\begin{array}{cc}
h_{11} & h_{12} \\
h_{12}^{*} & -h_{11}^{*}
\end{array}\right), \quad \mathcal{M}_{1}^{*} \mathcal{M}_{1}=\left\|h_{1}\right\|^{2}
$$

with the same way;

$$
\begin{array}{r}
r_{2}=\mathcal{M}_{2} s+N_{2}, \quad \mathcal{M}_{2}=\left(\begin{array}{lr}
h_{21} & h_{22} \\
h_{22}^{*} & -h_{21}^{*}
\end{array}\right), \\
\mathcal{M}_{2}^{*} \mathcal{M}_{2}=\left\|h_{2}\right\|^{2} I_{2} \quad(21)
\end{array}
$$

Thereby, the decision variable $b_{l}$ at the $\mathrm{l}^{\text {th }}$ antenna is;

$$
b_{l}=\mathcal{M}_{l}^{*}\left(\mathcal{M}_{l} s+N_{l}\right)=\left\|h_{l}\right\|^{2} s+\mathcal{M}_{l}^{*} N_{l}
$$

That means;

$$
\begin{aligned}
& b_{1}=\left\|h_{1}\right\|^{2} s+\mathcal{M}_{1}^{*} N_{1} \\
& b_{2}=\left\|h_{2}\right\|^{2} s+\mathcal{M}_{2}^{*} N_{2}
\end{aligned}
$$

The MRC decision can be expressed as the normalised sum

of b1 and b2. For the channel tap vector $h_{2}=$

$\left(h_{21} h_{22} h_{21} h_{22}\right)^{t}$ we have;

$$
\begin{gathered}
b=\frac{1}{\|h\|}\left(b_{1}+b_{2}\right)=\frac{\left\|h_{1}\right\|^{2}+\left\|h_{2}\right\|^{2}}{\|h\|} s \\
+\frac{1}{\|h\|}\left(\mathcal{M}_{1}^{*} N_{1}+\mathcal{M}_{2}^{*} N_{2}\right)
\end{gathered}
$$

while $\left\|h_{1}\right\|^{2}+\left\|h_{2}\right\|^{2}=\left|h_{11}\right|^{2}+\left|h_{22}\right|^{2}+\left|h_{21}\right|^{2}+\left|h_{12}\right|^{2}$, the MRC decision vector can be presented by;

$$
b=\|h\| s+N
$$

where the noise vector is $N=\frac{\mathcal{M}_{1}^{*} N_{1}+\mathcal{M}_{2}^{*} N_{2}}{\|h\|}$, the covariance of noise $\mathrm{N}$ is given by;

$$
E\left(N N^{*}\right)=\mathcal{M}_{1}^{*} E\left(N_{1} N_{1}^{*}\right) \mathcal{M}_{1}+\mathcal{M}_{2}^{*} E\left(N_{2} N_{2}^{*}\right) \mathcal{M}_{2}
$$

Since $E\left(N_{l} N_{l}^{*}\right)=2 \sigma^{2} I_{2}, l=1,2$, we get;

$$
E\left(N N^{*}\right)=2 \sigma^{2} \frac{\left\|h_{1}\right\|^{2}+\left\|h_{2}\right\|^{2}}{\|h\|^{2}} I_{2}=2 \sigma^{2} I_{2}
$$

\section{Minimum Mean Square Error Combining}

MRC and MMSE are two standard combining techniques. The combining weights for the MRC scheme are calculated by considering the communication channel, the noise and the interference power at the receiver. The combining weights for MMSE combining are determined based on the communication channel and the spatial noise and interference covariance matrix (Winters, 1984). Thus, when the noise is spatially correlated and added to the signal, MRC can't be optimal technique. In this case, the optimal combining technique is in the MMSE. To achieve high performance at the receivers, the weights of the MMSE combining technique are calculated by;

$$
w^{*}=\arg \min E\left\{\left(w^{t} r-s\right)^{2}\right\}
$$

So the optimal weight $w^{*}$ can be written as;

$$
\mathrm{w}^{*}=\mathrm{R}^{-1} \mathrm{~h}^{*}
$$


where $\mathrm{R}$ becomes the correlation noise and interferences. In the absence of interference, $\mathrm{R}=\mathrm{E}\left(\mathrm{NN}^{*}\right)$. Furthermore, for $2 \times 2$ MIMO system, and white noise, $\mathrm{E}\left(\mathrm{NN}^{*}\right)=\sigma^{2} \mathrm{I}_{2}, \mathrm{MMSE}$ can be viewed as MRC up to a scaling factor.

\section{EGC Receiver}

The EGC compensate the phase rotations for each $\mathrm{y}_{\mathrm{j}}\left(\mathrm{k}^{\prime}\right)$, the magnitudes remain unchanged. This method has little less complex than the MRC. The combined signal for EGC technique is still the linear combination of the signals received from altered paths. The EGC receiver fixes the weights as $\mathrm{w}_{\mathrm{n}}=\mathrm{e}^{-\mathrm{j} \varphi \mathrm{n}}$, where $\varphi_{\mathrm{n}}$ is a uniformly distributed random phase process (Oestges \& Clerckx, 2007). This technique brings all phases of the received signal to a common point to combine them (Annamalai et al., 1999). As a result, the amplitude weights will be the same. MRC outperforms EGC, although EGC is in general easier and cheaper to implement than MRC (Praho et al., 2010). However, EGC is not often analysed within the receivers operating in PLC channels. In this paper, we focus on evaluating the BER of MIMO-OFDM signals in the presence of EGC receiver over PLC channels. The output of the EGC combiner technique is assumed by Simon et al. (2005) to be;

$$
\mathrm{SNR}=\frac{\mathrm{E}_{\mathrm{S}}}{\mathrm{LN}_{0}} \sum_{\mathrm{i}=1}^{\mathrm{L}} \alpha_{\mathrm{l}}
$$

While $\mathrm{N}_{0}$ is the noise power, $\mathrm{L}$ is the number of paths, $\mathrm{E}_{\mathrm{S}}$ is the energy per symbol.

\section{RESULTS AND DISCUSSION}

\section{A. Measurements Results}

The development of PLC networks requires an accurate description of the channel transfer function, channel capacity and noise. To benefit from MIMO advantage for PLC, channel transfer function and impulsive noise measurements need to be made. We consider the topology in Figure3. We consider two cases where:

Case 1: Without electrical appliances (we consider only 4 plugs with no load connection, and 2 extension leads with a length of $1.5 \mathrm{~m})$.
Case 2: The electrical appliances connected to the network were two personal computers, and extension 2 leads openended, with no load connection, see Figure 3.

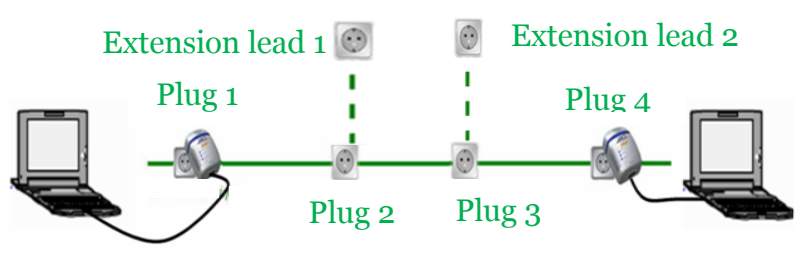

Figure 3. An experimental in-home PLC network

In this section, we analyse the performance of MIMOOFDM over PLC channel. Already discussed in (Dai, 2003), the principal challenge associated with the PLC network is the frequency selective fading channel, which performance degradation of the whole system. Therefore, transfer function of the PLC channel is one of the major challenges to establish the MIMO PLC system. For that reason, we perform first the measurements, using a Vector Network Analyzer, of the channel frequency response between for different feeding and receiving configurations. The measurements were carried out in the frequency band from 2 to $100 \mathrm{MHz}$. In a simulation, we exploit the MIMO PLC channels that have been measured. To do that, the bit error rate (BER) performance of MIMO-OFDM over MIMI-PLC channels with impulsive noise, is evaluated using computer simulation.

\section{MIMO-PLC channel mesurements}

To show the performance of MIM PLC system, it is crucial to study the PLC channel characteristics.

A typical 2×2 MIMO PLC transfer function measured over the experimental network is shown in Figure 4. From this figure, we can notice that, a strong destructive interference which is commonly referred to as a deep notch which affects the communication system negatively. Also, we observe that for these highly varying channels, there are several deep notches in the frequency response channel caused by the presence of the multiple electromagnetic paths in the PLC network. The other important aspect is that the cross-channel $\left(h_{12}, h_{21}\right)$ present deeper notches than co-channel $\left(h_{11}, h_{22}\right)$. We can explain this fact by the connection between different 
ports, the cross-channel depends on the coupling between different ports that mean there is no physical connection between transmitter and receiver, but for co-channels, the transmitter and receiver ports are physically connected. In wireless communication systems, this distinction between co-channel and cross channels is not required. However, this distinction is more important in case MIMO PLC realisation because a physical connection between receiver and transmitter is always present (Lars et al., 2014).

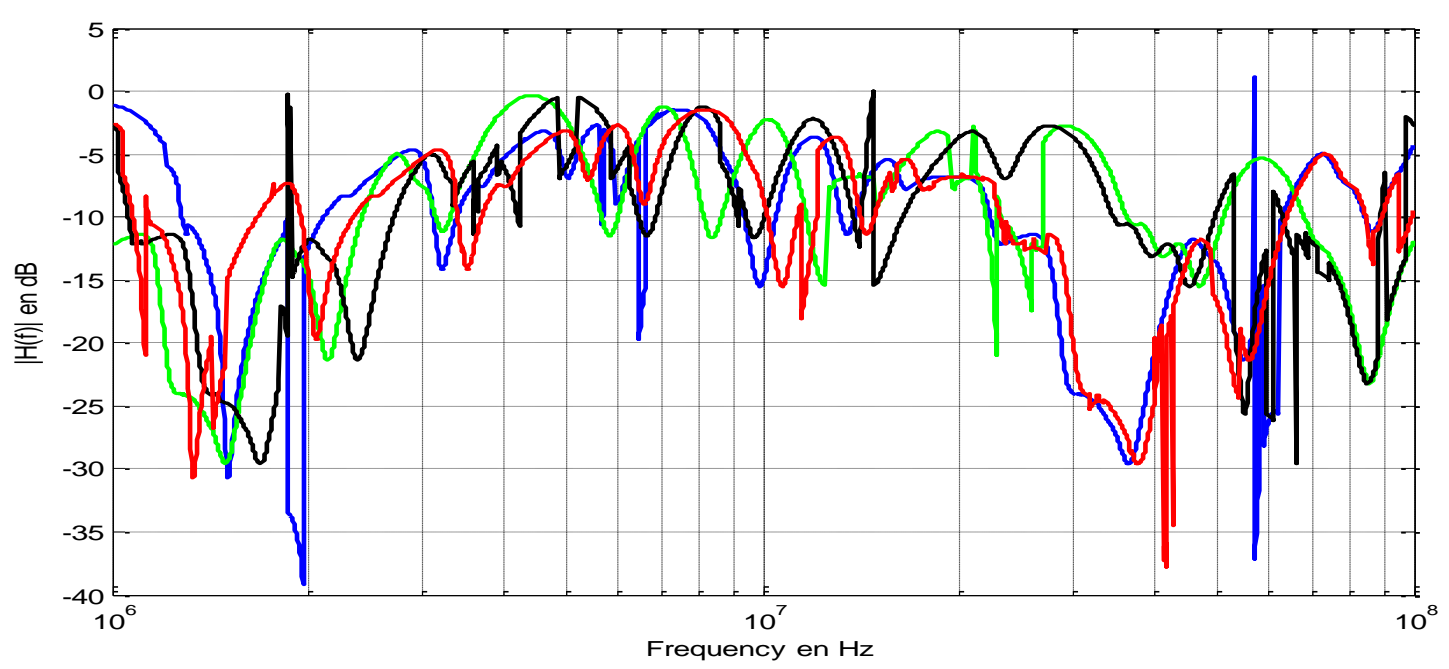

Figure 4. Experimental measurements of frequency response magnitude for MIMO-PLC channels (Case 1)

\section{Measured MIMO PLC channel noise}

To benefit from MIMO technology for the PLC network, impulsive noise measurements need to be made. Figure 5 shows the observed noise on an in-home PLC network. Noise measurement realised on $220 \mathrm{~V}$ using autonomous UPS. This Figure shows some decayed impulses. They have a vertical jumping at the start and reduce by the exponentiallydecaying-bound sinusoidal function. The pulse peak measured is up to $0.19 \mathrm{~V}$. The measured impulsive noise follows a Poisson process. It clear from Figure 5 that the level of P-N noise which corresponds to the physical connection between transmitter and receiver is lower than P-PE noise and the N-PE.

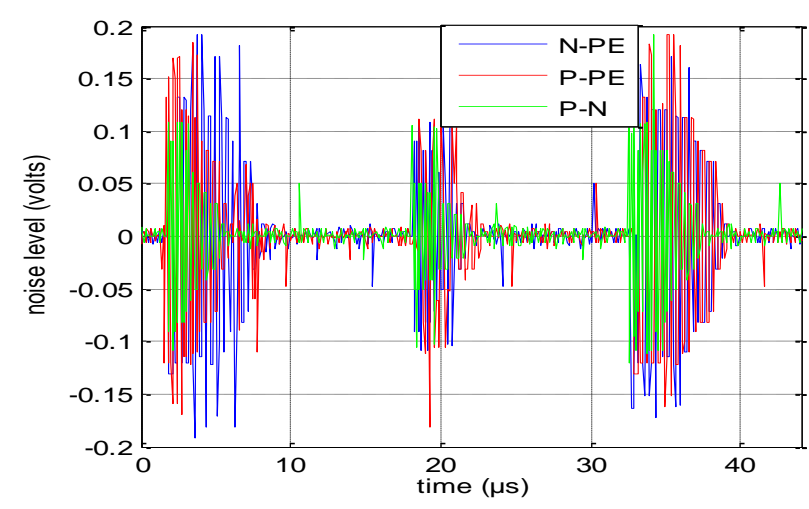

Figure 5. Typical measured MIMO PLC channel noise on an in-home PLC network

\section{The capacity of the PLC channel}

To measure the PLC channel capacity, we measure the channel capacity on a band of $24 \mathrm{kHz}$. This later band is used in a commercial OFDM based PLC technology as a carrier spacing. In this work, we focus on the 2-30 MHz and 2-100 $\mathrm{MHz}$ bands with a carrier spacing $\Delta \mathrm{f}$ of $24 \mathrm{kHz}$ in which the sub-channel can be treated as frequency-flat channels. For 2$30 \mathrm{MHz}$ band, only 1250 sub-channels were considered while 4083 sub-channels were considered for 2-100 $\mathrm{MHz}$ band. To satisfy CISPR requirements on radiated emissions (Praho et al., 2010), we use a power spectral density of $-50 \mathrm{dBm} / \mathrm{Hz}$ in 2-30 $\mathrm{MHz}$ band and $-80 \mathrm{dBm} / \mathrm{Hz}$ in 30-100 band.

The MIMO capacity $\mathrm{C}_{\text {MIMO }}$ can be presented by;

$$
\mathrm{C}_{\text {MIMO }}=\sum_{\mathrm{k}=1}^{\mathrm{l}} \Delta \mathrm{f} \sum_{\mathrm{i}}^{\mathrm{n}} \log _{2}\left(1+\frac{\mathrm{P}_{\mathrm{T}}\left(\mathrm{f}_{\mathrm{k}}\right) \lambda_{\mathrm{i}}\left(\mathrm{f}_{\mathrm{k}}\right)}{\mathrm{N}_{\mathrm{R}}\left(\mathrm{f}_{\mathrm{k}}\right) \mathrm{l}}\right) \text { bits. sec }
$$

where $N_{R}\left(f_{k}\right)$ is the power of coloured Gaussian noise, $P_{T}\left(f_{k}\right)$ is the PSD, $\lambda_{i}\left(f_{n}\right)$ is the eigenvalues of $H H^{H}$ where $H(f)$ is the frequency response of the PLC channel and $(.)^{\mathbf{H}}$ represents the matrix Hermitian. The SISO capacity is calculated by;

$$
\mathrm{C}_{\text {SISO }}=\sum_{\mathrm{k}=1}^{1} \Delta \mathrm{flog}_{2}\left(1+\frac{\mathrm{P}_{\mathrm{T}}\left(\mathrm{f}_{\mathrm{k}}\right) \lambda_{\mathrm{i}}\left(\mathrm{f}_{\mathrm{k}}\right)}{\mathrm{N}_{\mathrm{R}}\left(\mathrm{f}_{\mathrm{k}}\right)}\right) \text { bits. sec }
$$


The average spectral efficiency can be presented as the ratio between the average value of the channel capacity and the correspondent transmission bandwidth;

$$
\eta=\frac{\mathrm{C}_{\text {mean }}}{\mathrm{N} \Delta \mathrm{f}}
$$

\section{i. MIMO capacity of measured PLC channel}

To measure the capacity along the PLC channel, we measure the capacity on a small band of $24 \mathrm{kHz}$. Then, we can make the sum of all the bands on bands considered (30 MHz or 100 $\mathrm{MHz})$.

In the purpose of taken advantage of MIMO techniques for PLC network, channel capacity needs to be made. $2 \times 2$ MIMO capacity versus frequency is shown in Figure 6. We can notice that MIMO capacity is lower for $30-100 \mathrm{MHz}$ band, which corresponds to a lower transmit power. The capacity of $2 \times 2$ MIMO in the band 2-30 MHz is better than for the band 30$100 \mathrm{MHz}$

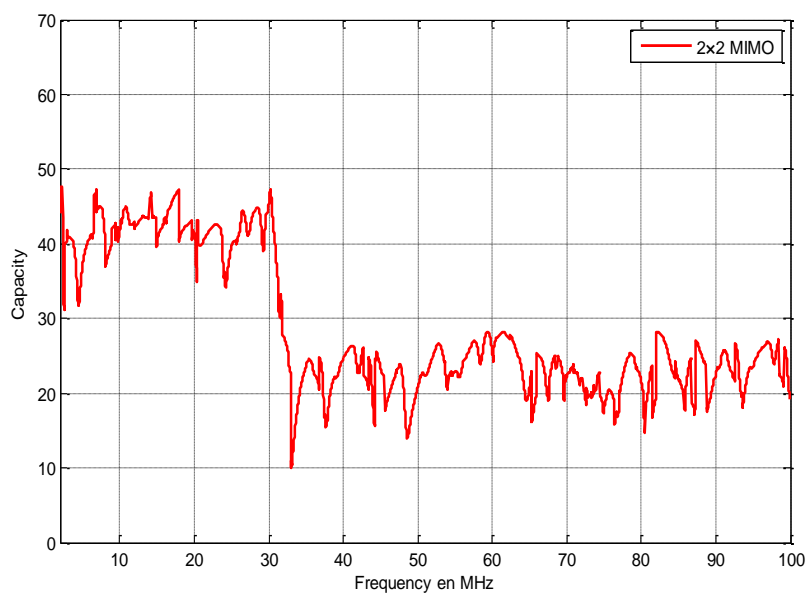

Figure 6. 2×2 MIMO capacity

\section{ii. Impact of electrical appliances on channel capacity}

Electrical appliances linked or not linked to the electrical grid at any time cause changes in the PLC network. Let us see the influence of Electrical appliances on the PLC channel capacity. To determine the influence of Electrical appliances, the power-line configuration with Electrical appliances was considered in Figure 3 (case 2). In Table 1, we report the min, mean, maximum and average spectral efficiency $\eta$ value of

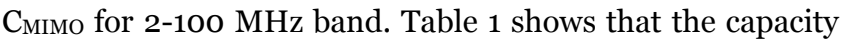
decreases when Electrical appliances are connected to the network
Table 1. Min, Mean and Max values of measured MIMO channel capacity and average spectral efficiency $\eta$

\begin{tabular}{lcccr}
\hline & $\begin{array}{l}\text { Min\{C\} } \\
(\mathrm{Gbps})\end{array}$ & $\begin{array}{l}\text { Mean\{C\} } \\
(\mathrm{Gbps})\end{array}$ & $\begin{array}{l}\operatorname{Max}\{\mathrm{C}\} \\
(\mathrm{Gbps})\end{array}$ & $\begin{array}{l}\boldsymbol{\eta} \\
(\mathrm{bps} / \mathrm{Hz})\end{array}$ \\
\hline Without & 2.09 & 2.69 & 3.21 & 27.45 \\
Elec. App & & & & \\
With & 1.61 & 2.15 & 2.89 & 21.94 \\
Elec. App & & & & \\
\hline
\end{tabular}

iii. MIMO capacity of random PLC channel

In Table 2, we report the min, mean, and maximum value of $\mathrm{C}_{\text {MIMo }}$ and $\mathrm{C}_{\text {sIso }}$ for two transmission bands. Furthermore, we compute the average spectral efficiency $\eta$.

Table 2. Min, mean and max values of measured MIMO channel capacity and average spectral efficiency $\eta$

\begin{tabular}{|c|c|c|c|c|c|}
\hline & $\begin{array}{l}\text { Band } \\
(\mathrm{MHz})\end{array}$ & $\begin{array}{l}\operatorname{Min}\{\mathrm{C}\} \\
(\mathrm{Gbps})\end{array}$ & $\begin{array}{l}\operatorname{mean}\{\mathrm{C}\} \\
(\mathrm{Gbps})\end{array}$ & $\begin{array}{l}\operatorname{Max}\{C\} \\
\text { (Gbps) }\end{array}$ & $\begin{array}{l}\boldsymbol{\eta} \\
(\mathrm{bps} / \mathrm{Hz})\end{array}$ \\
\hline \multirow[t]{2}{*}{ SISO } & $2-30$ & 0.12 & 0.38 & 0.51 & 13.57 \\
\hline & $2-100$ & 0.37 & 1.27 & 1.71 & 12.95 \\
\hline \multirow[t]{2}{*}{ MIMO } & $2-30$ & 0.21 & 0.76 & 1.09 & 27.14 \\
\hline & $2-100$ & 0.68 & 2.48 & 3.55 & $25 \cdot 31$ \\
\hline
\end{tabular}

We notice that average spectral efficiency $\eta$ of MIMO and SISO is higher in the band ranging from 2 to $30 \mathrm{MHz}$. It is important to notice that the average increase of the achievable rate is not proportional to the increase in the transmission bandwidth.

\section{B. Simulations Results}

In this section, via computer simulation, we show the performance of the proposed system under various PLC channel conditions. The multi-path random channel $h(t)$ is simulated using the Zimmermann channel model (Zimmerman et al., 2002). Inter symbols interferences (ISI) is an important factor constraining BER performance of MIMO-OFDM high-speed PLC communication systems. MMSE, EGC and MRC receivers can be used to eliminate ISI. In (Qian et al., 2019), impulse noise was analysed under difference mitigation schemes. In this work, we evaluate the proposed system when the impulse noise is considered. To do that, we use Middleton's class A model which is a suitable 
impulsive noise model for typical PLC (Mathur et al., 2015). Space-Time coding. The goal of the next simulations is to To model PLC channel noise, we use the Middleton class A model (Middleton, 1977), whose pdf (probability density function) is given by;

$$
\begin{aligned}
& P_{A}(n)=\sum_{m=0}^{\infty} \frac{e^{-A} A^{m}}{m !} \frac{1}{\sigma_{m} \sqrt{2 \pi}} \exp \left(-\frac{n^{2}}{2 \sigma_{m}^{2}}\right) \\
& \sigma_{m}^{2}=\sigma^{2} \frac{\frac{m}{A}+\Gamma}{\Gamma+1}
\end{aligned}
$$

where $\mathrm{m}$ is the number of active impulses, $\mathrm{A}$ is the impulsive index and defines the degree of impulsiveness. $\Gamma=\frac{\sigma_{\mathrm{g}}^{2}}{\sigma_{\mathrm{I}}^{2}}$ is the ratio of the variance of the impulse noise $\sigma_{\mathrm{I}}^{2}$ and the variance of the background noise $\sigma_{\mathrm{g}}^{2}$. if $\Gamma \rightarrow \infty$, the Middleton's Class A noise model degenerate to a Gaussian distribution. $\sigma^{2}=\sigma_{\mathrm{g}}^{2}+$ $\sigma_{\mathrm{I}}^{2}$ is the total noise power.

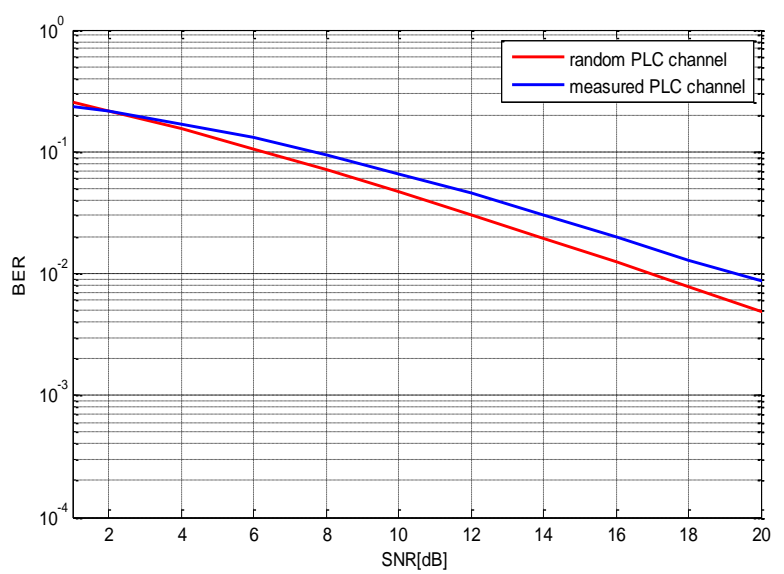

Figure 7. BER of MIMO-OFDM for random and measured PLC channels. $(\mathrm{N}=512,16-\mathrm{PSK})$ explore the benefits of channel diversity in PLC networks.

In the next simulation, we study the diversity gain for a variable number of transmitting and/or receiving antennas, $1 \times j=2 \times 2,2 \times 1$ and SISO, see Figure 8 . For $2 \times 1$ and $2 \times 2$ systems, the diversity gains in signal to noise (SNR) over SISO system are $2 \mathrm{~dB}$ and $14 \mathrm{~dB}$ for BER $=10^{-2}$. The $2 \times 2$ system performance is $11 \mathrm{~dB}$ better than that of the $2 \times 1$ system. So, we can conclude that: thanks to the full transmitter diversity gain of MISO ( $2 \times 1$ configuration), MISO system outperforms SISO system. Also, thanks to the full transceiver diversity gain of MIMO ( $2 \times 2$ configuration), MIMO system outperform MISO system.

This is reasonable because as the number of receivers increases the performance should improve.

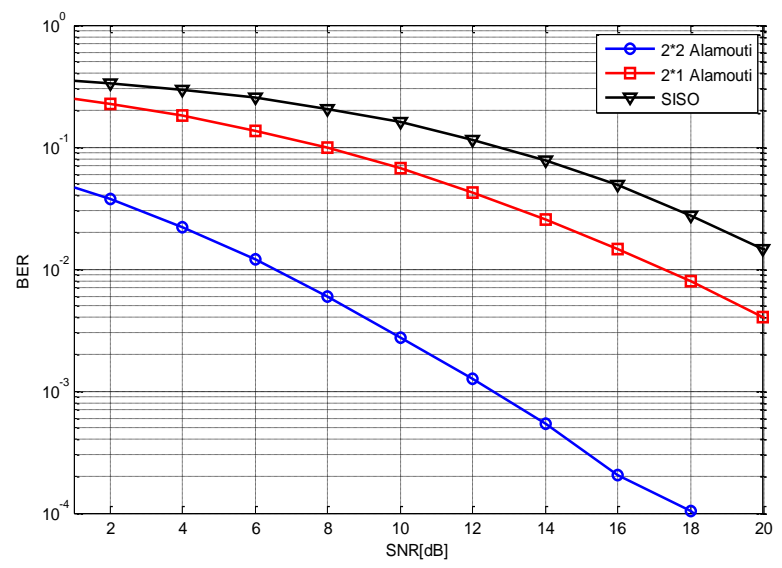

Figure 8. BER of MIMO-OFDM PLC systems. $(\mathrm{N}=512,16-$ PSK)

In our investigation, we take $\mathrm{A}=0.1$ and $\Gamma=0.1$, implying that the impulsive noise power is 10 higher than the white noise. The OFDM parameters are carrier spacing equal to 24 $\mathrm{kHz}$, the number of subcarriers is 2048. The channel is assumed as being known at the receiver. We use 16-PSK and 32-PSK constellations. At the same time, the performances of MMSE, EGC and MRC receivers are also simulated for comparison.

From Figure 7, a slight improvement in term of BER is reached when a random channel is used. Diversity has a specific sense related to a random channel. Thus, channel diversity can be exploited through a random channel. Here we have the underlying stochastic model for the PLC channel. If the channel is not random, diversity does not make sense. MIMO technique can still reach spatial diversity by using
In Figure 9, we compare the BED of 16-PSK and 32-PSK with different receiver methods in MIMO-OFDM. MMSE combining technique achieves better performance over the whole range of SNRs. From Figure 9, Over the region odB $\leq$ SNR $\leq 6 \mathrm{~dB}$, it is shown that the MRC scheme performs a little better than the EGC receiver and slightly the same that of the MMSE. The performance of EGC is about $1 \mathrm{~dB}$ worse than MRC, with somewhat less complexity. So, MRC receiver can operate at low SNR. However, for SNR more than $10 \mathrm{~dB}$, the EGC receiver performs better than the MRC receiver system. Thus, compared to the MMSE scheme, EGC scheme can slightly remove ISI to a certain degree. But strong ISI makes this effect strictly restricted. The MMSE scheme proposed in this paper utilises channel state information to select strongest path signals We see that, at high SNR, the use of 
MMSE receiver gives respectively $1 \mathrm{~dB}$ and $3 \mathrm{~dB}$ performance improvements than EGC receiver and the MRC receiver.

In the presence of multi-path effect and impulsive noise, MRC receiver performs much worse than the others receiver except at very low SNR. In this zone, EGC performs much worst due to noise amplification. So, it is very clear from the results that MMSE is suitable for MIMO-PLC system. MMSE not only excludes ISI components but also minimises the total power of noise which is the factor that affects PLC technology the most.

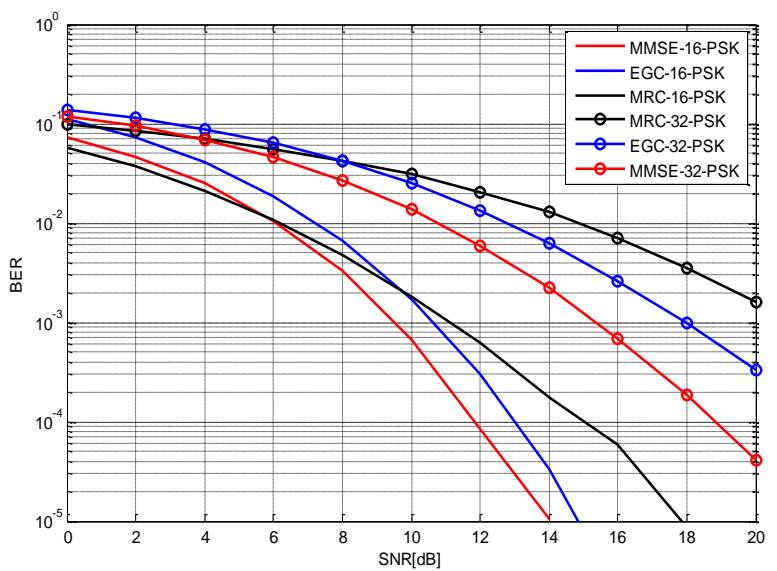

Figure 9. BER comparison of MMSE, EGC, and MRC based 2×2 MIMO-OFDM PLC system

\section{CONCLUSION}

In this work, we confirmed that Broadband MIMO PLC systems with high bandwidth efficiencies are feasible for PLC environments. The MIMO PLC channel characteristics were presented based on a measurement campaign which was performed on a specific electrical network. The effect of the electrical appliances on the capacity of a MIMO PLC system with two transmits ports, and two receiver ports were presented. It is observed that electrical appliances decrease the MIMO channel capacity. In addition, we prove that MMSE outperform MRC and EGC receivers in a MIMO-PLC system in the presence of impulsive noise. The measurements and simulation results carried out in this work demonstrated that the MIMO system could achieve a notable enhancement in overall system performance.

\section{REFERENCES}

A, Annamalai, C, Tellambura \& VK, Bhargava, 1999, 'Exact Evaluation of Maximal Ratio and Equal-Gain Diversity Receivers for M-ary QAM on Nakagami Fading Channels', IEEE Trans. on Communications, Vol. 47, pp. 1335-1344. 10.1109/26.789669

A, Mathur, MR, Bhatnagar \& BK, Panigrahi, 2015, 'Performance Evaluation of PLC Under the Combined Effect of Background and Impulsive Noises', IEEE Communications Letters, vol. 19, no. 7, pp.11171120. 10.1109/LCOMM.2015.2429129

B, Praho, M, Tlich, P, Pagani, A, Zeddam, \& F, Nouvel, 2010, 'Cognitive Detection Method of Radio Frequencies on Power Line Networks', in Proc. IEEE Int. Symp. Power Line Commun. and Its App. (ISPLC), pp. 225-230.

C, Yoon, H, Lee, 2012, Efficient soft-output demapping method for MIMO-OFDM WLAN systems, in Future
Information Technology, Application, and Service, Lecture Notes in Electrical Engineering, vol. 164, pp. 485-491.

C, Oestges \& B, Clerckx, 2007, MIMO Wireless, Communication from real world propagation to spacetime code design, Academic Press, 1 edition.

D, Middleton, 1977, 'Statistical-physical model of electromagnetic interference', IEEE Trans. Electromagn. Compat., vol. EMC-19, no. 3, pp. 106-126. 10.1109/TEMC.1977.303527

Ertugrul 2016, 'On multiple-input multiple-output OFDM with index modulation for next generation wireless networks', IEEE Transactions on Signal Processing, vol. 64 no.15, pp. 3868-3878. 10.1109/TSP.2016.2551687

H, Dai, HV, 2003, Advanced signal processing for power line communications, IEEE Commun. Mag. Vol. 41, No. 5, 2003, pp.100-107. 10.1109/MCOM.2003.1200108 
J, Mestoui et al., 2019, 'Performance analysis of CE-OFDM-

CPM Modulation using MIMO system over wireless channel', $J$ Ambient Intell Human Comput. https://doi.org/10.1007/s12652-019-01628-o

$\mathrm{JH}$, Winters 1984, 'Optimum combining in digital mobile radio with co-channel interference', IEEE Transactions on Vehicular Technology, vol. 33, no. 3, 528-539.

Llano, D. De La Vega, I, Angulo \& L. Marron 2019, 'Impact of Channel Disturbances on Current Narrowband Power Line Communications and Lessons to Be Learnt for the Future Technologies', IEEE Access, vol. 7, pp. 83797-83811. 10.1109/ACCESS.2019.2924806

MK, Simon \& M-S, Alouini 2005, Digital Communication over Fading Channels, 2nd ed. New Jersey, USA: John Wiley \& Sons.

N, Shlezinger, R, Shaked \& R, Dabora 2018, 'On the Capacity of MIMO Broadband Power Line Communications Channels', IEEE Transactions on Communications, vol. 66, no. 10, pp. 4795-4810. 10.1109/TCOMM.2018.2835472

TB, Lars, A, Schwager, P, Pagani \& DM, Schneider 2014, MIMO Power Line Communications: Narrow and Broadband Standards, EMC and Advanced Processing, Taylor \& Francis Group, LLC.

Y, Qian, X, Zhou, J, Li, F, Shu \& DNK, Jayakody 2019, 'A Novel Precoding and Impulsive Noise Mitigation Scheme for MIMO Power Line Communication Systems', IEEE Systems Journal, vol. 13, no. 1, pp. 617. 10.1109/JSYST.2018.2880962

Zimmerman, M, Dostert, K 2002, 'A multi-path model for the power-line channel', IEEE Trans. Commun. vol. 50, no. 4, pp. 553-559._10.1109/26.996069 